\title{
Impaired glucose phosphorylation and transport in skeletal muscle cause insulin resistance in HIV-1-infected patients with lipodystrophy
}

\author{
Georg M.N. Behrens, ${ }^{1}$ Anne-Rose Boerner, ${ }^{2}$ Klaus Weber, ${ }^{1}$ Joerg van den Hoff,,3 \\ Johann Ockenga, ${ }^{4}$ Georg Brabant,${ }^{5}$ and Reinhold E. Schmidt ${ }^{1}$ \\ ${ }^{1}$ Department of Clinical Immunology, \\ ${ }^{2}$ Department of Nuclear Medicine, Hannover Medical School, Hannover, Germany \\ ${ }^{3}$ PET Center Rossendorf, Dresden, Germany \\ ${ }^{4}$ Department of Gastroenterology and Hepatology, and \\ ${ }^{5}$ Department of Clinical Endocrinology, Hannover Medical School, Hannover, Germany
}

\begin{abstract}
Insulin resistance is a frequently observed side effect of highly active antiretroviral therapy (HAART). Currently, very little is known about the mechanisms or specific tissues involved. We aimed to identify possible defects in skeletal muscle glucose uptake and metabolism in HIV patients receiving HAART. Whole-body glucose disposal and oxidation were determined by combination of the euglycemic-hyperinsulinemic clamp technique and indirect calorimetry. Muscle glucose uptake of the thighs was measured simultaneously by dynamic $2\left[{ }^{18} \mathrm{~F}\right]$ fluoro-2-deoxy-D-glucose positron emission tomography. Patients receiving HAART had signs of lipodystrophy as confirmed by dual energy $\mathrm{x}$-ray absorptiometry. Whole-body glucose disposal was significantly reduced in these patients compared with untreated patients. Analysis of kinetic constants using a three-compartment model indicated reduced skeletal glucose uptake caused by significantly impaired glucose transport and phosphorylation. Skeletal muscle glucose uptake was reduced by $66 \%$ in treated patients and explained $46 \%$ and $43 \%$ of whole-body glucose disposal in patients on HAART and therapy-naive patients, respectively. Insulin-stimulated whole-body oxidative and nonoxidative glucose disposal was significantly lower in the treated group, as was suppressive insulin action on lipolysis. To our knowledge, this is the first report providing in vivo evidence that, in lipodystrophic HIV patients, impaired glucose transport and phosphorylation cause reduced insulin-mediated glucose uptake.
\end{abstract}

J. Clin. Invest. 110:1319-1327 (2002). doi:10.1172/JCI200215626.

\section{Introduction}

A syndrome of lipodystrophy (peripheral fat loss and/or abdominal obesity) and hyperlipidemia has been reported in up to $60-80 \%$ of patients treated with highly active antiretroviral therapy (HAART) $(1,2)$. Hyperinsulinemia, increased C-peptide concentration, insulin resistance, and impaired glucose tolerance have been frequently observed $(3,4)$. Insulin resistance, as determined by euglycemic-hyperinsulinemic clamp technique, reflects defective insulin action observed predominantly in skeletal muscle and liver. This is a

Received for publication April 8, 2002, and accepted in revised form August 28, 2002.

Address correspondence to: Georg M.N. Behrens, Immunology Division, The Walter and Eliza Hall Institute of Medical Research, Post Office of The Royal Melbourne Hospital, Parkville 3050, Victoria, Australia. Phone: 61-3-9345-2482; Fax: 61-3-9347-0852; E-mail: Behrens@wehi.edu.au.

Conflict of interest: No conflict of interest has been declared. Nonstandard abbreviations used: highly active antiretroviral therapy (HAART); positron emission tomography (PET); $2\left[{ }^{18} \mathrm{~F}\right]$ fluoro-2-deoxy-D-glucose (F-18-FDG); bioelectrical impedance analysis (BIA); dual energy $x$-ray absorptiometry (DEXA); fat-free mass (FFM); phosphorylation fraction (PF); homeostasis model assessment (HOMA); whole-body glucose uptake $(\mathrm{M})$; nucleoside-analogue reverse transcriptase inhibitor (NRTI). critical feature of the metabolic syndrome, a chronic and progressive disorder frequently leading to diabetes mellitus type 2 . The potential development of diabetes mellitus, hyperlipidemia, and possible cardiovascular events associated with HAART is of great concern (5). Accordingly, long-term toxicities have an important impact on decisions to initiate treatment, composition of first-line therapy, and patient adherence.

The heterogeneity of the lipodystrophy syndrome and the observation that almost all antiretroviral drugs have been linked with its development appear to suggest a multifactorial pathogenesis. A direct role for protease inhibitors in inducing insulin resistance is suggested by several observations: (a) rapid development of insulin resistance after initiation of protease inhibitor therapy (indinavir) in HIV-infected patients and even healthy volunteers (6-8), (b) improvement of insulin sensitivity after substitution of nevirapine or abacavir for protease inhibitors (9), (c) reversal of hyperglycemia following withdrawal of protease inhibitor, (d) hyperinsulinemia prior to the occurrence of body composition changes and hyperlipidemia (10), and (e) reduced insulin-stimulated glucose uptake by adipocytes and muscle cells in vitro upon treatment with protease inhibitors $(11,12)$.

Glucose transport is a critical step for insulin-stimulated glucose utilization in skeletal muscle, which is a 
potentially important site of insulin resistance. It has been difficult to assess the in vivo rates of insulin-stimulated glucose uptake and phosphorylation in humans. The "glucose clamp" method determines insulin sensitivity but does not separate glucose transport and phosphorylation from subsequent steps of metabolism. Therefore, we have employed positron emission tomography (PET) imaging of skeletal muscle uptake of a short-lived tracer, $2\left[{ }^{18} \mathrm{~F}\right]$ fluoro-2-deoxyD-glucose (F-18-FDG). This provides a technology for analogous in vivo assessment of glucose transport and phosphorylation $(13,14)$.

The current study was undertaken to test the hypothesis that impaired glucose transport and/or phosphorylation within the skeletal muscle contribute to the insulin resistance in HIV patients that develop lipodystrophy during HAART. We performed dynamic PET imaging of mid-thigh muscle F-18-FDG uptake during insulin-stimulated conditions, together with indirect calorimetry in both therapy-naive patients and HAART-treated patients with signs of lipodystrophy. Our findings provide the first in vivo evidence, to our knowledge, that impaired glucose transport together with impaired glucose phosphorylation significantly accounts for insulin resistance in patients with lipodystrophy receiving HAART.

\section{Methods}

Patients. Six male HIV-infected patients receiving HAART including a protease inhibitor (three receiving zidovudine plus 3TC, one d4T plus ddI, and two d4T and 3TC; two receiving ritonavir plus indinavir, one ritonavir plus saquinavir, one saquinavir, one nelfinavir, and one lopinavir/ritonavir) were compared with six male therapy-naive HIV patients. Both groups' physical and disease-related characteristics are presented in Table 1 . None of the patients received other medication known to affect insulin sensitivity. The nature, purpose, and potential risks of the study were explained to all subjects before they gave their written informed consent to participate. The study was approved by the Ethical Committee of the Hannover Medical School.

Body composition analysis. Body composition analysis included anthropometric measurements, bioelectrical impedance analysis (BIA), dual energy $\mathrm{x}$-ray absorptiometry (DEXA), and 24-hour urinary creatinine and urea excretion analysis. Whole-body muscle mass was calculated from arm muscle area according to the method of Heymsfield et al. (15) and from creatinine excretion assuming that $1 \mathrm{~g}$ creatinine $=18.5 \mathrm{~kg}$ muscle (three consecutive 24-hour urine collections) (13). DEXA (Lunar DPX-IQ; Lunar Corp., Cologne, Germany) was used to measure total body fat, truncal and abdominal fat, and fat-free mass (FFM). BIA included assessment of total body water, fat, and FFM by a radiofrequency current of $800 \mu \mathrm{A}$ at $50 \mathrm{kHz}$ (RJL Systems Inc., Detroit, Michigan, USA) as described (16).

Experimental design. All patients received an isocaloric diet (approximately $30 \mathrm{cal} / \mathrm{kg} / \mathrm{d}$; carbohydrate/protein/ fat $50 \% / 15 \% / 35 \%$ ) for 3 days before PET examination.
All patients receiving HAART were advised to take their antiretroviral drugs as they normally did. Hence, medication was taken 1-3 hours before the start of the clamp. Following a 12-hour overnight fast, the indirect calorimetry was initiated (minute -50 ), a normoglycemic hyperinsulinemic clamp was performed (0 minutes), and the dynamic PET scan was started at 90 minutes. Two peripheral venous catheters were inserted, one into an antecubital vein for the infusion of test substances, and the other retrogradely into a wrist vein for blood sampling. The hand was then placed in a box heated at $50-60^{\circ} \mathrm{C}$ to achieve arterialization of the blood. The subjects were lying supine, were asked to remain motionless and awake, and were allowed 30 minutes to equilibrate to the environment.

Respiratory gas exchange measurements. Respiratory gas exchange measurements were performed using a ventilation hood open circuit indirect calorimeter (Deltatrac Metabolic Monitor; Datex Instruments Inc., Helsinki, Finland) (16). Gas exchange analysis was started at -50 minutes and continued until the end of the study at 150 minutes. Indirect calorimetry data were calculated as mean values during the last 30 minutes of both periods: basal, -30 to 0 minutes, and clamp, 120-150 minutes. Substrate oxidation rates were calculated according to previously published methods $(13,16)$. Rates of protein oxidation were used to determine the nonprotein respiratory quotient (NPRQ). Glucose and lipid oxidation rates were determined based on an NPRQ of 0.707 for $100 \%$ fat oxidation and 1.00 for $100 \%$ glucose oxidation. Protein oxidation was calculated from the urinary urea excretion after correction for changes in the urea pool (17), considering the urea distribution volume equivalent to the total body water from BIA assessment.

Whole-body glucose uptake. Whole-body glucose uptake was determined using the euglycemic-hyperinsulinemic clamp technique (insulin infusion rate 1 $\mathrm{mU} / \mathrm{kg} / \mathrm{min}$ ) performed as previously described from 0 to 150 minutes $(13,18)$. Blood glucose was monitored every 5 minutes during the insulin infusion, and euglycemia was maintained throughout the clamp by infusing $20 \%$ dextrose at a variable rate. The steady state for arterialized plasma glucose concentrations was always reached and maintained after 90 minutes. Calculation of whole-body glucose metabolism (glucose disposal) was based on the glucose infusion rate after correction for changes in the glucose space (18). The metabolic clearance rate for insulin was computed as described by DeFronzo et al. (18). Glucose oxidation was calculated from respiratory gas exchange data, and nonoxidative glucose metabolism was taken as the difference between glucose disposal and glucose oxidation. All data were normalized for $\mathrm{kg}$ of FFM.

PET and data analysis. Subjects were positioned in an ECAT Exact 922 PET scanner (CTI Molecular Imaging Inc., Knoxville, Tennessee, USA/ Siemens Medical Systems Inc., Hoffman Estate, Illinois, USA) (reconstructed image resolution $7 \mathrm{~mm}$ [full width at half-maximum], $16 \mathrm{~cm}$ transaxial field of view) with the mid-thigh corre- 
sponding to the scanner's axial field of view. A 30-minute transmission scan was collected to measure individual attenuation coefficients, which were applied during reconstitution of images for quantitative mapping of radioactivity. Dynamic F-18-FDG PET imaging comprised 12 frames at 10 -second intervals, 12 frames at 15 seconds, 10 frames at 30 seconds, 4 frames at 5 minutes, and 3 frames at 10 minutes. A blood sample per time frame was collected during dynamic scanning for measurement of plasma radioactivity withdrawn during dynamic scanning. A three-compartment model for F18-FDG tissue activity in skeletal muscle was used to derive rate constants of F-18-FDG uptake, transport, and phosphorylation (Figure 1) $(13,14)$.

According to this model, the first-order rate constant, $k_{1}(\mathrm{ml} / \mathrm{min} / \mathrm{ml})$, reflects clearance of F-18-FDG from blood into the tissue compartment that includes both intracellular and interstitial space. The rate constant $k_{2}$ $\left(\mathrm{min}^{-1}\right)$ cannot be attributed to a single defined process like glucose transport out of the muscle. $k_{2}$ rather represents outward transport of free (nonphosphorylated) F-18-FDG from the intracellular space back into plasma. It may also reflect returning F-18-FDG by diffusion to the plasma from the interstitial space, because it has not entered into the myocyte. The rate constants $k_{3}\left(\mathrm{~min}^{-1}\right)$ and $k_{4}\left(\mathrm{~min}^{-1}\right)$ refer, respectively, to the phosphorylation of F-18-FDG via the hexokinase reaction and dephosphorylation of F-18-FDG-6-P via the glucose-6-phosphatase reaction.

Arterialized blood time-activity curves were used as an input function. The model assumes that F-18-FDG is administered in trace amounts, glucose metabolism in tissue is in steady state, the transport of F-18-FDG and F-18-FDG-6-P between compartments has firstorder kinetics, and plasma glucose concentration is constant. Rates of glucose utilization $\left(k_{3}\right)$ can be estimated using modeling from dynamic data. In this work it was further assumed that $k_{4}$ was negligible over the duration of the study since phosphorylated F-18FDG is not further metabolized to any appreciable extent due to low amounts of glucose-6-phosphatase. The local metabolic rate $(K)$ of F-18-FDG is calculated from the relationship of the individual rate constants according to the equation $K=\left(k_{1} k_{3}\right) /\left(k_{2}+k_{3}\right)$. The local metabolic rate $K(\mathrm{ml} / \mathrm{min} / \mathrm{ml})$ for F-18-FDG is related to glucose consumption by a factor termed the lumped constant (LC) as the denominator. The LC is the ratio of analog (F-18-FDG) to true glucose. Recent studies $(19,20)$ estimated the LC to be 1.2 for skeletal muscle in healthy volunteers and obese or type 2 diabetic subjects during euglycemic insulin-stimulated conditions and revealed that the LC appears not to be as sensitive to insulin stimulation or resistance as glucose transport is $\left(k_{1}\right)$.

We calculated two additional parameters. The ratio $k_{1} / k_{2}$ corresponds to the distribution volume of free F-18-FDG $\left(\mathrm{DV}_{\mathrm{CE}}\right)$ and reflects glucose transport. Because the amount of free glucose in the intracellular space is regarded as very small compared with that in the interstitial space, an elevated value of $k_{2}$ may reflect free F-18-FDG that has entered the interstitial space from plasma but has not entered the intracellular space. Accordingly, impaired glucose transport may be reflected by reduced $k_{1}$ and increased $k_{2}$. The phosphorylation fraction (PF) of F-18-FDG $\left[\mathrm{PF}=k_{3} /\left(k_{2}+k_{3}\right)\right]$ reflects the disposition for nonphosphorylated F-18FDG within the tissue compartment to be phosphorylated or to return from the tissue compartment back to the plasma compartment. It has a theoretical range of values from 0 to 1 . PF can be interpreted as an index of the extent to which glucose phosphorylation serves as the rate-limiting step of glucose metabolization compared with glucose transport. The decrease of $\mathrm{PF}$ toward a value of 0 reflects a situation where phosphorylation is rate-limiting $(20,21)$.

Other measurements. Arterialized venous blood samples were taken at basal ( 0 minutes) and after 120 minutes for determination of the serum or plasma concentration of insulin, C-peptide, and proinsulin, and after 140 minutes for urea, FFAs, acetate, and $\beta$-hydroxybutyrate. Oral glucose tolerance (OGT) test was performed as described recently (3) on a separate day. Fasting glucose and insulin levels from the day of OGT and PET study were used to calculate insulin resistance index by the homeostasis model assessment (HOMA) (22). The concentrations of insulin and C-peptide were measured by commercially available RIAs (Insulin RIA from Pharmacia Diagnostics Inc., Freiburg, Germany; C-peptide RIA from Diasorin Inc., Düsseldorf, Germany). Lipoproteins (LDL, HDL, VLDL) and apolipoproteins (apoA-I, apoA-II, apoB, apoE, and lipoprotein [a]) were determined as described recently using nephelometric quantification (3). Nonesterified FFAs were measured by an enzymatic assay (NEFA C; Wako Chemicals Inc., Neuss, Germany). Soluble TNF- $\alpha$ receptors were analyzed using specific immunoassays as described elsewhere (23). CD4 lymphocyte cell count was determined by flow cytometry and HIV-RNA copies by quantitative PCR (Amplicor HIV-1 Monitor Test; Roche Diagnostic Systems, Branchburg, New Jersey, USA). All patients had total and free testosterone,

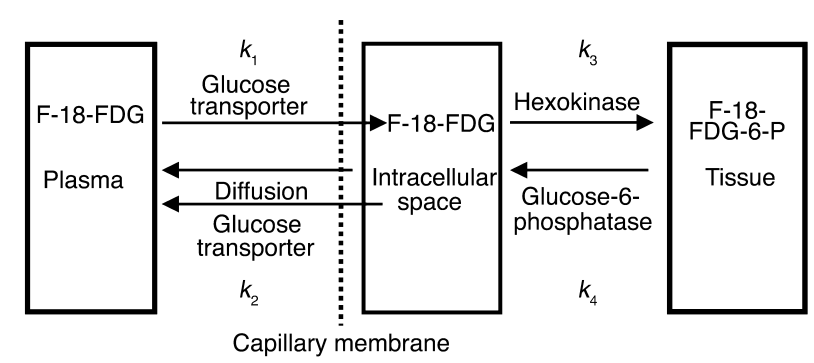

\section{Figure 1}

Three-compartment model for the F-18-FDG kinetics of the PET data. Dynamically acquired PET data and arterial plasma time course of F-18-FDG activity were used as input functions in the compartmental modeling. The rate constants represent inward transport $\left(k_{1}\right)$, outward transport $\left(k_{2}\right)$, and phosphorylation $\left(k_{3}\right)$ of F-18-FDG. 
Table 1

Clinical data, body composition, and insulin resistance parameters of the patients

\begin{tabular}{|c|c|c|c|}
\hline & Patients on therapy & Therapy-naive patients & $P$ value \\
\hline Age (years) & $51.3 \pm 4.7$ & $43.5 \pm 3.9$ & NS \\
\hline Weight (kg) & $70.1 \pm 2.6$ & $75.6 \pm 4.8$ & NS \\
\hline $\operatorname{BMI}\left(\mathrm{kg} / \mathrm{m}^{2}\right)$ & $23.9 \pm 0.7$ & $22.9 \pm 1.1$ & NS \\
\hline Viral load $\left(\log _{10} \mathrm{HIV}-\mathrm{RNA} / \mathrm{ml}\right)$ & $2.43 \pm 0.34$ & $5.16 \pm 0.39$ & $<0.001$ \\
\hline CD4 lymphocytes (cells/ $\mu \mathrm{l})$ & $444 \pm 66$ & $336 \pm 38$ & NS \\
\hline Duration of HIV infection (months) & $113 \pm 23$ & $16 \pm 11$ & 0.004 \\
\hline Antiretroviral therapy (months) & $61.7 \pm 5.5$ & - & \\
\hline Protease inhibitor (months) & $44.2 \pm 4.2$ & - & \\
\hline Truncal fat $(\mathrm{kg})$ (\% of total fat) & $5.61 \pm 0.44(67 \%)$ & $6.98 \pm 1.30(52 \%)^{A}$ & NS \\
\hline Extremity fat ( $\mathrm{kg}$ ) (\% of total fat) & $1.98 \pm 0.19(23 \%)$ & $5.15 \pm 0.89(40 \%)^{\mathrm{A}}$ & 0.015 \\
\hline Percent truncal fat/Percent extremity fat & $2.9 \pm 0.2$ & $1.3 \pm 0.1$ & $<0.001$ \\
\hline Total body fat (kg) (\% of total weight) & $7.6 \pm 0.6(11 \%)$ & $12.0 \pm 2.1(16 \%)$ & NS \\
\hline Fat-free mass (kg) & $59.7 \pm 2.6$ & $59.3 \pm 4.0$ & NS \\
\hline Waist-to-hip-ratio & $1.08 \pm 0.1$ & $0.9 \pm 0.01$ & 0.01 \\
\hline Triceps skinfold thickness ( $\mathrm{mm}$ ) & $4.8 \pm 0.7$ & $10.7 \pm 2.3$ & 0.02 \\
\hline Biceps skinfold thickness (mm) & $3.7 \pm 0.7$ & $7.5 \pm 0.6$ & 0.002 \\
\hline HOMA IR & $7.3 \pm 1.3$ & $3.3 \pm 1$ & 0.037 \\
\hline MCR for insulin ( $\mathrm{ml} / \mathrm{min})$ & $941.6 \pm 186.7$ & $2061.9 \pm 251.4$ & 0.005 \\
\hline Insulin/Glucose (pmol/mmol) & $33 \pm 7$ & $17.7 \pm 5.1$ & NS \\
\hline sTNF receptor $1(\mathrm{pg} / \mathrm{ml})$ & $1,331.8 \pm 157.5$ & $1,223.6 \pm 202$ & NS \\
\hline sTNF receptor $2(\mathrm{pg} / \mathrm{ml})$ & $3,687 \pm 340.2$ & $3,679.5 \pm 545.3$ & NS \\
\hline
\end{tabular}

Data are presented as mean \pm SEM. HOMA IR, insulin resistance index determined according to homeostasis model assessment (22). MCR, metabolic clearance rate (22); NS, not significant. ${ }^{A} P<0.001$ for comparison of percentages between the two groups.

24-hour urinary cortisol excretion, and thyroid parameters within normal values.

Statistical analysis. All data are presented as means \pm SEM. The differences between treated and untreated patients were analyzed by unpaired $t$ test or Mann-Whitney $U$ test, where appropriate. The Wilcoxon test was used for paired variables (basal and clamp). Correlation of two values was determined by Pearson's coefficient. Statistical calculations were performed using SPSS 10.0.7 (SPSS Science, Chicago, Illinois, USA). $P$ values less than 0.05 were considered to indicate statistical significance.

\section{Results}

Body composition. Both patient groups had comparable mean total body fat mass and mean FFM as determined by DEXA and BIA. Patients on HAART, however, showed evidence for lipodystrophy with peripheral fat loss and relative increase in abdominal fat mass, assessed by DEXA and confirmed by anthropometric parameters (Table 1). HAART was associated with mixed hyperlipidemia and significantly increased values for triglycerides, VLDL, apoB, and apoE (data not shown) in concordance with earlier observations $(3,24)$.

Insulin sensitivity. Patients with or without treatment had comparable fasting glucose concentrations (mean $5.32 \pm 0.5 \mathrm{mmol} / \mathrm{l}$ vs. $4.65 \pm 0.3 \mathrm{mmol} / \mathrm{l}, P=0.33$ ). Euglycemia was maintained during the insulin infusion, with mean glucose levels of $5.3 \pm 0.19 \mathrm{mmol} / \mathrm{l}$ vs. $4.69 \pm 0.24 \mathrm{mmol} / 1$ in treated and untreated subjects, respectively. During insulin infusion, plasma insulin increased in the treated group from basal $229.3 \pm 44.3$ $\mathrm{pmol} / \mathrm{l}$ to $606.3 \pm 164.3 \mathrm{pmol} / \mathrm{l}$, and, in the therapy-naive group, from basal $131.5 \pm 41.6 \mathrm{pmol} / 1$ to $248.8 \pm 23.4$ pmol/1 ( $P=0.002$ between groups). The metabolic clearance rate for insulin was significantly reduced in patients on HAART ( $P=0.005$; Table 1$)$. C-peptide concentrations decreased in both groups during the clamp, as expected. Patients on HAART had significantly lower rates of insulin-stimulated whole-body glucose uptake (M) compared with therapy-naive patients $(14.2 \pm 0.5$ $\mu \mathrm{mol} / \mathrm{kg} / \mathrm{min}$ vs. $32.2 \pm 4.0 \mu \mathrm{mol} / \mathrm{kg} / \mathrm{min}, P=0.001$; Figure 2). In line with the clamp data, the HOMA insulin resistance index was significantly different between the groups (Table 1). Across the entire study population, HOMA insulin resistance index and M were correlated with the percent truncal to percent extremity fat ratio $(r=0.61, P=0.034$, and $r=-0.69$, $P=0.013$, respectively). In therapy-naive patients the oxidative glucose metabolism during clamp conditions increased significantly $(11.7 \pm 2.4 \mu \mathrm{mol} / \mathrm{kg} / \mathrm{min})$ compared with basal conditions $(5.0 \pm 1.3 \mu \mathrm{mol} / \mathrm{kg} / \mathrm{min}$, $P=0.028)$ and was higher compared with that of patients on treatment $(5.3 \pm 1.33 \mu \mathrm{mol} / \mathrm{kg} / \mathrm{min}, P=0.042)$ (Figure 3). In contrast, patients on HAART presented, during clamp, only a slight increase in oxidative glucose disposal (basal $4.0 \pm 0.9 \mu \mathrm{mol} / \mathrm{kg} / \mathrm{min}$ ) and a significantly lower nonoxidative glucose metabolization $(8.9 \pm 1.6 \mu \mathrm{mol} / \mathrm{kg} / \mathrm{min}$ vs. $20.3 \pm 4.0 \mu \mathrm{mol} / \mathrm{kg} / \mathrm{min}$; Figure 3). Importantly, when a correction was made considering the differences in whole-body glucose uptake, the percentage of oxidative and nonoxidative glucose metabolism of mean total glucose uptake during the clamp was identical in both groups: $37 \%$ was metabolized by oxidation and $63 \%$ was metabo- 

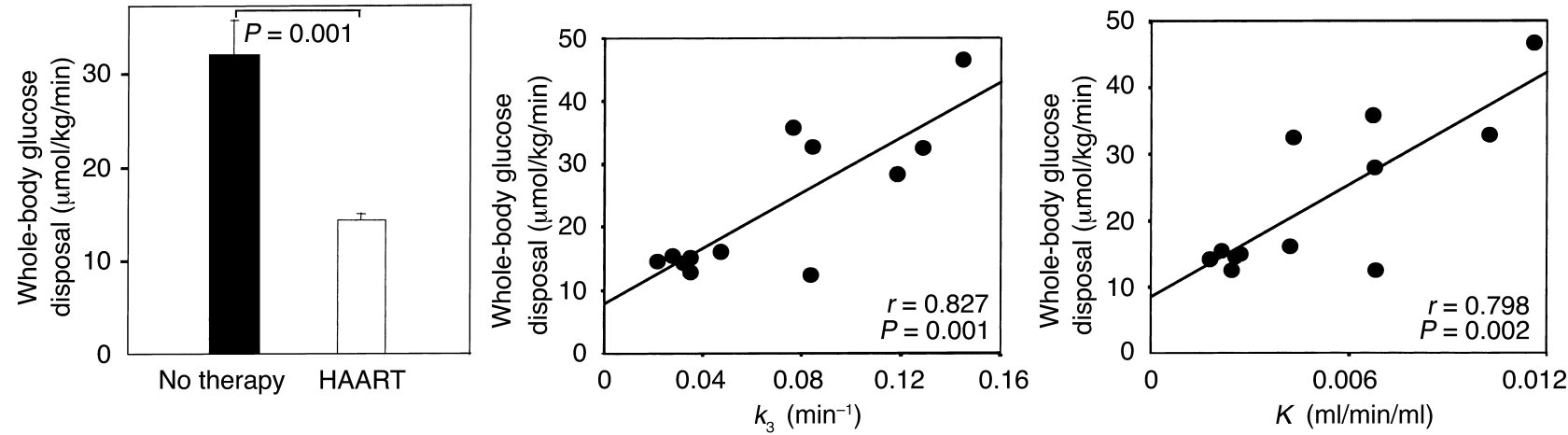

Figure 2

Whole-body glucose utilization during euglycemic-hyperinsulinemic clamp. The left panel represents the insulin-stimulated systemic glucose uptake (M) per kg FFM during clamp in both patient groups. The middle and right panels depict the correlation between whole-body glucose utilization and the rate constants $k_{3}$ (middle) and $K$ (right) for F-18-FDG uptake from three-compartment modeling. $K$ is a PET parameter of F-18-FDG clearance from blood to muscle.

lized by the nonoxidative pathway. Therefore, insulinstimulated total-body glucose disposal, total oxidative glucose disposal, and total nonoxidative glucose disposal were reduced by approximately $50 \%$ in the patients on HAART.

PET study. Uptake of F-18-FDG into the mid-thigh muscle $(K)$ was significantly lower in the group receiving HAART $(0.00311 \pm 0.00077 \mathrm{ml} / \mathrm{min} / \mathrm{ml}$ vs. 0.00730 $\pm 0.00123 \mathrm{ml} / \mathrm{min} / \mathrm{ml}, P=0.015) . K$ is calculated from the rate constants $k_{1}, k_{2}$, and $k_{3}$, which respectively represent inward transport, outward transport, and phosphorylation of F-18-FDG. The rate constants for both groups are presented in Figure 4 . There was no difference in the rate constants for inward transport $\left(k_{1}\right)$ into skeletal muscle. During insulin infusion, however, the rate constant for phosphorylation of glucose $\left(k_{3}\right)$ was significantly reduced in patients receiving HAART $\left(0.03875 \pm 0.00923 \mathrm{~min}^{-1}\right.$ vs. $0.09964 \pm 0.01508 \mathrm{~min}^{-1}$, $P=0.016$ ), whereas the rate constant $k_{2}$ (back diffusion from interstitial space/back transport from intracellular space) was higher in these patients. Across all patients, whole-body glucose uptake was significantly correlated with $K$ and $k_{3}$ (Figure 2). The values for $\mathrm{DV}_{\mathrm{CE}}$ $(0.0946 \pm 0.0058 \mathrm{ml} / \mathrm{ml}$ vs. $0.1391 \pm 0.0156 \mathrm{ml} / \mathrm{ml}$, $P=0.041)$ and $P F(0.1589 \pm 0.0358$ vs. $0.4384 \pm 0.0615$, $P=0.004)$ were each significantly higher in therapynaive patients than in patients on HAART (Figure 5). The correlation between whole-body glucose uptake $(\mathrm{M})$ and PF was highly significant $(r=0.78, P=0.003)$. Finally, muscle glucose uptake calculated from PET data and from anthropometric or urine creatinine excretion measurements revealed that the contribution of whole-body muscle glucose uptake to whole-body glucose uptake was $46 \%$ (range $30-110 \%$ ) and $43 \%$ (range $28-60 \%$ ) in patients on HAART and therapynaive patients, respectively. Skeletal muscle glucose uptake was reduced by $66 \%$ in patients receiving HAART compared with untreated patients $(1.25 \pm 0.29$ $\mu \mathrm{mol} / 100 \mathrm{~g}$ muscle per minute vs. $2.82 \pm 0.48$ $\mu \mathrm{mol} / 100 \mathrm{~g}$ muscle per minute, $P=0.02$ ).
Indirect calorimetry. Basal $\mathrm{VO}_{2}$ was $274.7 \pm 12.5 \mathrm{ml} /$ min in the group receiving HAART and $276.6 \pm 20.5$ $\mathrm{ml} / \mathrm{min}$ in therapy-naive patients. Basal respiratory quotient $(R Q)$ was $0.78 \pm 0.01$ and $0.78 \pm 0.02$, respectively. In the last 30 minutes of the clamp, RQ increased significantly to $0.85 \pm 0.2(P=0.023)$ in the therapynaive group compared with the group receiving HAART, which had an unchanged mean RQ. The mean basal energy expenditure (patients on HAART, 1,839.6 \pm 85.1 $\mathrm{kcal} / \mathrm{d}, 119 \% \pm 2 \%$ Harris-Benedict prediction [HB]; vs. therapy-naive patients, $1,862.7 \pm 136.1 \mathrm{kcal} / \mathrm{d}$, $108 \% \pm 5 \% \mathrm{HB}$ ) remained stable during insulin-infusion in patients receiving HAART and decreased slightly in therapy-naive patients $(1,791.6 \pm 124.9 \mathrm{kcal} / \mathrm{d}$, $104 \% \pm 4 \% \mathrm{HB}, P=0.007$ between groups). Patients on HAART had comparable rates of fat oxidation during basal and clamp conditions $(1.2 \pm 0.27 \mathrm{mg} / \mathrm{kg} / \mathrm{min}$ vs. $1.14 \pm 0.07 \mathrm{mg} / \mathrm{kg} / \mathrm{min}$ ), whereas therapy-naive patients showed reduced fat oxidation, from basal $1.3 \pm 0.19$ $\mathrm{mg} / \mathrm{kg} / \mathrm{min}$ to $0.65 \pm 0.18 \mathrm{mg} / \mathrm{kg} / \mathrm{min}$ during insulin infusion $(P=0.028$ within the group; $P=0.03$ between groups during clamp).
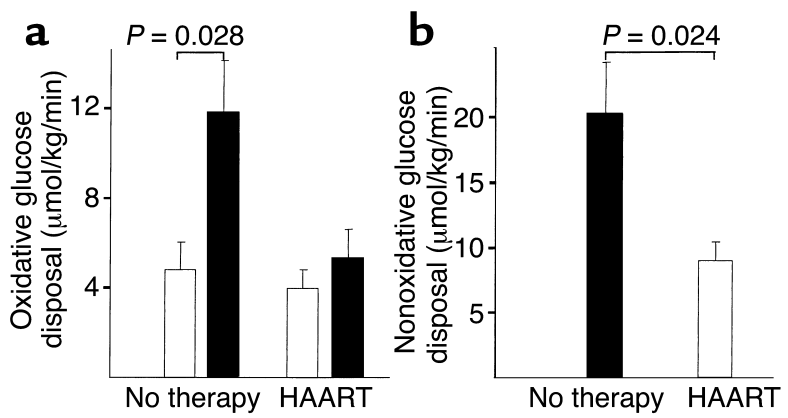

Figure 3

Influence of insulin infusion on glucose metabolization assessed by indirect calorimetry. (a) Glucose oxidation, basal (white bars) and during clamp conditions (black bars), in untreated HIV patients and patients on HAART. (b) Nonoxidative glucose metabolism during clamp in both study groups. 


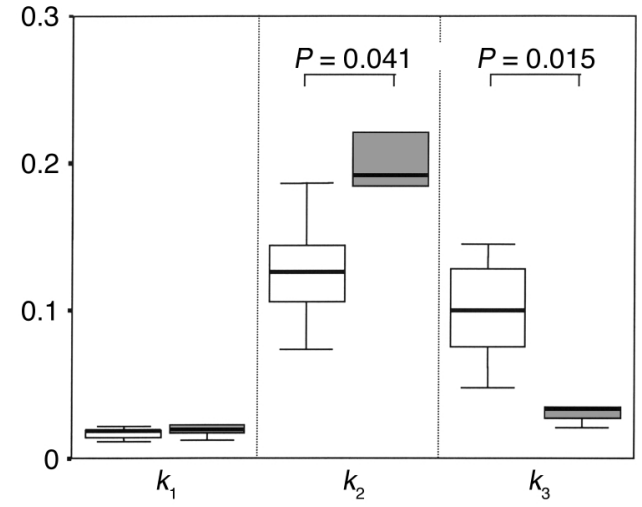

Figure 4

Rate constants from three-compartment modeling of dynamic PET for F-18-FDG metabolism. Comparison of therapy-naive patients (white regions) and patients on HAART (gray regions). $k_{1}$ $(\mathrm{ml} / \mathrm{min} / \mathrm{ml})$ and $k_{2}\left(\mathrm{~min}^{-1}\right)$ represent the inward and outward transport, respectively, from the extracellular into the intracellular compartment. $k_{3}\left(\mathrm{~min}^{-1}\right)$ is the rate constant for the phosphorylation of F-18-FDG. Data are median and interquartile range; the bars represent the complete range. $P$ values represent results of Mann-Whitney statistics between groups.

To address the role of other factors known to contribute to insulin resistance and predicted to be important in HIV-infected individuals, we determined levels of FFAs and the activation status of the TNF system. During basal conditions, FFAs were comparable in both groups, but insulin-mediated suppression of FFAs was clearly diminished in patients on HAART compared with untreated patients (Table 2). Ketone bodies decreased significantly only in the therapy-naive group. Like the insulin resistance parameters (HOMA index and $\mathrm{M}$ ), the insulin-mediated suppression of lipolysis as indicated by FFAs concentration during clamp was significantly associated with the percent truncal to percent extremity fat ratio $(r=0.826, P=0.001)$. Finally, the soluble TNF receptor analysis revealed no difference in levels of both soluble TNF receptor 1 (sTNFR1) and sTNFR2 (Table 1).

\section{Discussion}

HAART has significantly improved the survival and prognosis of HIV-infected individuals. However, until now, side effects such as lipodystrophy and metabolic disturbances have not been pathogenetically elucidated. The current study reveals several important new observations regarding the major site and mechanisms of insulin resistance during antiretroviral therapy. Using PET, we provide direct in vivo evidence that impairment of glucose transport and phosphorylation contributes significantly to impaired systemic glucose uptake in patients receiving HAART. In addition, our study demonstrates the skeletal muscle as a primary site of impaired peripheral glucose utilization. The total insulin-stimulated glucose disposal in patients receiving HAART was less than $50 \%$ of that in the therapy-naive control group. At the same time, whole-body glucose oxidative disposal and nonoxidative disposal were depressed to a similar extent. These data extend recent in vitro studies $(11,12,25,26)$ proposing impairment of intrinsic GLUT4 activity or inhibition of GLUT4 translocation following incubation with indinavir, resulting in impaired glucose transport, as a major cause for insulin resistance. The role of glucose phosphorylation, however, has not yet been assessed, and the differences between in vitro and in vivo results may depend on different tissues, drugs and drug combinations, pharmacokinetic conditions, and secondary factors like FFA and lipodystrophy. We hypothesize that acute insulin resistance induced by protease inhibitors in HIV patients may mainly depend on primary drug-related mechanisms (8). This condition is later augmented and maintained by more complex abnormalities of the lipid metabolism, the development of peripheral fat loss and central obesity, and mitochondrial toxicity induced by nucleoside reverse transcriptase inhibitors. As a result, impaired glucose transport and glucose phosphorylation contribute to insulin resistance in patients with lipodystrophy receiving HAART. Interestingly, lipodystrophic patients presented with markedly elevated resting energy expenditure and decreased insulin-induced thermogenesis during clamp; this presentation resembles known abnormalities observed in individuals with insulin resistance and diabetes. The reduction or delay in thermogenesis in the control group was quite unexpected and needs to be confirmed in further studies. Minor insulin resistance, already increased basal resting energy expenditure, and HIV-induced mitochondrial damage are possible factors explaining this observation.

The question remains, where and how is skeletal muscle glucose phosphorylation affected in lipodystrophic patients? With respect to the proportions of the total glucose disposal that are oxidative or nonoxidative metabolized, patients on HAART presented with $50 \%$ reduced disposals of both pathways. Thus, we speculate that glucose phosphorylation is inhibited immediately following glucose uptake in the muscle cell, affecting both metabolic pathways to a similar
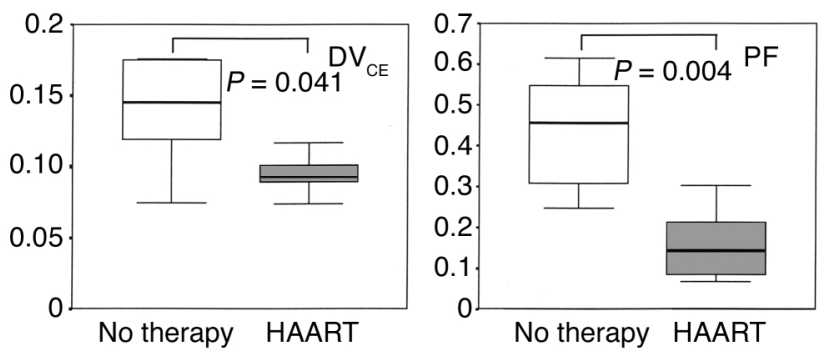

\section{Figure 5}

Differences in the distribution volume ( $\left.\mathrm{DV}_{\mathrm{CE}}\right)$ and the PF of F-18-FDG metabolism. DV $V_{C E}$ (left panel) reflects glucose transport, and $\mathrm{PF}$ (right panel) quantitatively describes the relative influence of glucose phosphorylation on the overall rate of glucose utilization. Both parameters were significantly higher in therapy-naive patients (white regions) than in patients with lipodystrophy on HAART (gray regions). 


\section{Table 2}

Insulin-induced changes in selected metabolic parameters in both patient groups

\begin{tabular}{lcc} 
& Basal & Clamp \\
& & \\
Patients on HAART & & \\
Glucose $(\mathrm{mmol} / \mathrm{l})$ & $5.32 \pm 0.5$ & $5.3 \pm 0.19$ \\
C-peptide $(\mathrm{ng} / \mathrm{ml})$ & $3.6 \pm 0.3^{\mathrm{A}}$ & $2.6 \pm 0.6^{\mathrm{A}, \mathrm{B}}$ \\
Proinsulin $(\mathrm{pmol} / \mathrm{l})$ & $41 \pm 14.7$ & $23.3 \pm 7.7$ \\
FFA $(\mathrm{mmol} / \mathrm{l})$ & $0.61 \pm 0.09$ & $1.01 \pm 0.23^{\mathrm{A}}$ \\
Acetone $(\mathrm{mg} / \mathrm{l})$ & $2.6 \pm 0.39$ & $2.4 \pm 0.3^{\mathrm{A}}$ \\
$\beta$-Hydroxybutyrate $(\mu \mathrm{mol} / \mathrm{l})$ & $158 \pm 23$ & $116.8 \pm 29.3$ \\
Therapy-naive patients & & \\
Glucose $(\mathrm{mmol} / \mathrm{l})$ & $4.65 \pm 0.3$ & $4.69 \pm 0.24$ \\
C-peptide $(\mathrm{ng} / \mathrm{ml})$ & $1.8 \pm 0.1$ & $1.3 \pm 0.1^{\mathrm{B}}$ \\
Proinsulin $(\mathrm{pmol} / \mathrm{l})$ & $22.2 \pm 10.5$ & $19 \pm 8.2$ \\
FFA $(\mathrm{mmol} / \mathrm{l})$ & $0.85 \pm 0.3$ & $0.38 \pm 0.1$ \\
Acetone $(\mathrm{mg} / \mathrm{l})$ & $4.1 \pm 1.6$ & $1.4 \pm 0.14^{\mathrm{B}}$ \\
$\beta$-Hydroxybutyrate $(\mu \mathrm{mol} / \mathrm{l})$ & $194.8 \pm 50.9$ & $70.3 \pm 6.2^{\mathrm{B}}$ \\
\hline AP 0.05 between groups, ${ }^{\mathrm{B} P}<0.05$ within groups (Wilcoxon test).
\end{tabular}

extent. Mitochondrial dysfunction and impaired oxidative capacity (especially in skeletal muscle) caused by nucleoside-analogue reverse transcriptase inhibitors (NRTIs) are well-known side effects of antiretroviral therapy (27-29). These mechanisms could also account for an impaired hexokinase activity in patients receiving HAART. Given that hexokinase, as an ATP-requiring enzyme, might have privileged access to ATP produced by oxidative phosphorylation, and that binding of hexokinase isoenzymes to mitochondria has been considered as a potential mechanism for the regulation of enzyme activity and glucose metabolism (30), the NRTI-related mitochondrial toxicity could be of particular importance. Mitochondrial damage by NRTI, for example, could reduce the amount of mitochondria-bound hexokinase and increase the level of free hexokinase with considerably higher sensitivity to inhibition by glucose-6-phosphate. Alternatively, impaired oxidative phosphorylation could limit the source and utilization of ATP in formation of glucose-6-phosphate (30). Impaired hexokinase activity and ATP production may also explain a synergistic effect of protease inhibitor-induced impairment of glucose transport and NRTI-associated mitochondrial dysfunction, leading to a higher incidence of lipodystrophy and insulin resistance in patients treated with a combination of NRTI and protease inhibitors $(31,32)$. Other direct drug-mediated events downstream of the glucose metabolization pathway, such as inhibition of citrate synthase activity by zidovudine, could facilitate this process (33). Further studies using nuclear magnetic resonance spectroscopy might be able to distinguish the mechanisms affecting the glucose transport, phosphorylation, oxidation, and storage in more detail.

The finding that skeletal muscle is the main site of insulin resistance in patients on HAART does not exclude a pathogenetic role for other tissues. The patients described here were hyperlipidemic and clearly demonstrated signs of abnormal fat redistribution (peripheral fat loss and central obesity) confirmed by DEXA and anthropometric methods. Also, the elimination of the insulin-mediated suppression of lipolysis and fat oxidation, even in the presence of considerably higher insulin levels in patients on HAART, clearly demonstrates impaired insulin action in this metabolic pathway. The molecular mechanism for this event remains to be determined. Increased intraabdominal fat, impaired insulin-mediated suppression of lipolysis, and perhaps protease inhibitor-induced lipolysis, as demonstrated in vitro $(11,34)$, can give rise to elevated concentrations of unesterified fatty acids (35). Consequently, these secondary factors may well play a pivotal role in the pathogenesis of the lipodystrophy syndrome and may explain several metabolic features of these patients. Increased FFA would lead to reduced glucose utilization by inhibition of carbohydrate oxidation, stimulate hepatic glucose production, and enhance insulin secretion. FFAs have also been suggested to impair insulin signaling and to reduce glucose transport activity, most likely altering GLUT4 trafficking or activity (36). Other authors provide evidence for an FFA-mediated proportional reduction of both glycogen synthesis and glycolysis by inhibition of glucose transport and/or phosphorylation, which could be relevant to this study (37). Even more importantly, FFA can have a direct effect on the hexokinase activity (38). Increased FFA may also explain the impaired insulin extraction by the liver (39), since patients on HAART presented with significantly reduced insulin clearance rates during clamp. Finally, there is solid evidence that FFA promoted gluconeogenesis, and regulation of endogenous glucose production is an important determinant of glucose tolerance and fasting glucose levels $(36,37,40)$. This is consistent with an association of lipodystrophy under HAART with reduced suppression of endogenous (hepatic) glucose production (41). The insulin concentrations achieved in the present study in the patients with lipodystrophy, however, are much higher than in reports by others (41) and should be sufficiently elevated to effectively decrease hepatic glucose production to negligible values. We cannot exclude a potential bias due to minor hepatic glucose production in the calculations of the whole-body glucose uptake. This, however, would not affect any of the conclusions based on the rate constants obtained by the PET.

Although they interfere with the interpretation of some group differences, high insulin levels are necessary to allow a correct calculation of the whole-body glucose uptake given the likely hepatic insulin resistance in patients with lipodystrophy (41). On the other hand, resulting group differences need to be considered in the interpretation of the study. One might question whether the considerable group differences in insulin levels during the clamp modulate the respective contributions of glucose transport and phosphorylation to systemic glucose uptake. A recent 
PET study by Williams et al. (20) revealed a dose-related insulin regulation of glucose transport and phosphorylation. The authors found that with high insulin levels during clamp, comparable to those in our patients on HAART, the reduced glucose phosphorylation in skeletal muscle was substantially corrected in both obesity and type 2 diabetes. The parameters of glucose transport that were corrected in obese subjects, however, remained reduced in type 2 diabetes. We calculated our data according to the same compartment model and, like Williams et al. (20), included the parameters $\mathrm{DV}_{\mathrm{CE}}$ and $\mathrm{PF}$, although these parameters have only infrequently been used and evaluated in muscle glucose metabolization. Surprisingly, reduced values for $k_{3}$ and $\mathrm{PF}$ in insulin-resistant HIV patients with signs of lipodystrophy suggest that impaired glucose phosphorylation contributes to insulin-stimulated glucose uptake even at marked levels of insulin stimulation. At the same time, despite comparable $k_{1}$ rate constants, our finding of a significantly reduced value for the transport parameter $\mathrm{DV}_{\mathrm{CE}}$ demonstrates that impaired glucose transport accounts substantially for insulin resistance as well.

The interpretation of data based on modeling techniques is limited by several factors. Optimal validation of the dynamic modeling and of the assumptions for the three-compartment four-rate constant model is difficult to achieve, and, consequently, resulting data do not necessarily provide ultimate answers. Possible bias could result from non-uniform distribution of material (e.g., F-18-FDG, hexokinase) in individual pools, use of a glucose analog instead of glucose, and mutual influences of rate constants employed to assess the role of glucose transport and phosphorylation in insulin resistance $(29,41)$. As an example, the PF and $\mathrm{DV}_{\mathrm{CE}}$ values are significantly influenced by the rate constant $k_{2}$, which represents a transport parameter. Thus, the differences in PF could reflect primarily transport rather than phosphorylation impairments. Values of $k_{3}$ and PF, however, were significantly correlated to whole-body glucose uptake, emphasizing an independent contribution of impaired glucose phosphorylation. We choose the three-compartment four-rate constant model for our calculations because it is currently the most commonly applied and most thoroughly evaluated model for measurements in skeletal muscle $(13,14,20,42,43)$.

A second limitation is that our study design cannot distinguish between the direct drug-induced mechanisms and the secondary events, like abnormal fat redistribution and increased FFAs, that lead to insulin resistance. All patients on therapy in this study received long-term treatment with different drug combinations, which confuses direct interpretation of causal relationships. Reduced insulin sensitivity has been reported in both healthy individuals and HIV-infected patients following short-term protease inhibitor therapy (indinavir) without signs of lipodystrophy, hypertriglyceridemia, or elevated FFA (6-8). We hypothesize that at least some of our results regarding impaired glucose transport and/or phosphorylation are induced directly by protease inhibitors.

There is some evidence for a role of cytokines like TNF- $\alpha$ in the development of both insulin resistance and HIV therapy-related lipodystrophy $(35,44)$. TNF$\alpha$ has multiple effects on adipocyte function, including inhibition of lipogenesis and increase of lipolysis. It has also been shown to impair insulin signaling and to reduce GLUT4 gene expression. Comparable levels of soluble TNF- $\alpha$ receptor levels across the entire cohort, however, are in line with results of the PET study and do not support a crucial role of TNF- $\alpha$ in insulin resistance in these patients.

In summary, we provide direct experimental evidence for impaired glucose transport and phosphorylation as major factors leading to reduced whole-body glucose uptake in patients receiving HAART and showing signs of lipodystrophy. At the same time, insulin-mediated suppression of lipolysis and fat oxidation is abolished in these patients. We conclude that in lipodystrophic patients receiving HAART, impairment of glucose uptake, oxidation, and storage is caused by a combination of primary drug-mediated and secondary metabolism-related mechanisms.

\section{Acknowledgments}

We gratefully acknowledge B. Zock, B. Woyte, R. Doering, and the staff of the PET laboratory for their excellent technical help. We also acknowledge R. Horn and M. Martin for technical assistance. This work was supported by a grant from the Hanover Medical School (HILF 79313003).

1. Carr, A., et al. 1999. Diagnosis, prediction, and natural course of HIV-1 protease inhibitor-associated lipodystrophy, hyperlipidaemia, and diabetes mellitus: a cohort study. Lancet. 353:2093-2099.

2. Behrens, G., Stoll, M., and Schmidt, R.E. 2000. Lipodystrophy syndrome in HIV infection: what is it, what causes it and how can it be managed? Drug Saf. 23:57-76.

3. Behrens, G., et al. 1999. Impaired glucose tolerance, beta cell function and lipid metabolism in HIV patients under treatment with protease inhibitors. AIDS. 13:F63-F70.

4. Walli, R., et al. 1998. Treatment with protease inhibitors associated with peripheral insulin resistance and impaired oral glucose tolerance in HIV1-infected patients. AIDS. 12:F167-F173.

5. Behrens, G., Schmidt, H., Meyer, D., Stoll, M., and Schmidt, R.E. 1998. Vascular complications associated with use of HIV protease inhibitors. Lancet. 351:1958.

6. Dube, M.P., et al. 2001. Prospective evaluation of the effect of initiating indinavir-based therapy on insulin sensitivity and B-cell function in HIVinfected patients. J. Acquir. Immune Defic. Syndr. 27:130-134.

7. Noor, M.A., et al. 2001. Metabolic effects of indinavir in healthy HIVseronegative men. AIDS. 15:F11-F18.

8. Noor, M.A., et al. 2002. Indinavir acutely inhibits insulin-stimulated glucose disposal in humans: a randomized, placebo-controlled study. AIDS. 16:F1-F6.

9. Martinez, E., Conget, I., Lozano, L., Casamitjana, R., and Gatell, J.M. 1999. Reversion of metabolic abnormalities after switching from HIV-1 protease inhibitors to nevirapine. AIDS. 13:805-810.

10. Mulligan, K., et al. 2000. Hyperlipidemia and insulin resistance are induced by protease inhibitors independent of changes in body composition in patients with HIV infection. J. Acquir. Immune Defic. Syndr. 23:35-43.

11. Rudich, A., et al. 2001. The HIV protease inhibitor nelfinavir induces insulin resistance and increases basal lipolysis in 3T3-L1 adipocytes. Diabetes. 50:1425-1431.

12. Nolte, L.A., et al. 2001. The HIV protease inhibitor indinavir decreases insulin- and contraction-stimulated glucose transport in skeletal muscle. Diabetes. 50:1397-1401. 
13. Selberg, O., et al. 1993. Insulin resistance in liver cirrhosis. Positron-emission tomography scan analysis of skeletal muscle glucose metabolism. J. Clin. Invest. 91:1897-1902.

14. Kelly, D.E., et al. 1996. The effect of non-insulin-dependent diabetes mellitus and obesity on glucose transport and phosphorylation in skeletal muscle. J. Clin. Invest. 97:2705-2713.

15. Heymsfield, S.B., McMannus, C., Smith, J., Stevens, V., and Noxo, D.W. 1982. Anthropometric measurements of muscle mass: revised equations for calculating bone-free arm muscle area. Am. J. Clin. Nutr. 36:680-690.

16. Suttmann, U., et al. 1993. Resting energy expenditure and weight loss in human immunodeficiency virus-infected patients. Metabolism. 42:1173-1179

17. Tappy, L., Owen, O.E., and Boden, G. 1988. Effect of hyperinsulinemia on urea pool size and substrate oxidation rates. Diabetes. 37:1212-1216.

18. DeFronzo, R.A., Tobin, J.D., and Andres, R. 1979. Glucose clamp technique: a method for quantifying insulin secretion and resistance. Am.J. Physiol. 237:E214-E223.

19. Peltoniemi, P., et al. 2000. Lumped constant for $\left[{ }^{18} \mathrm{~F}\right]$ fluordeoxyglucose in skeletal muscles of obese and non obese humans. Am. J. Physiol. Endocrinol. Metab. 279:E1122-E1130.

20. Williams, K.V., Price, J.C., and Kelley, D.E. 2001. Interaction of impaired glucose transport and phosphorylation in skeletal muscle and insulin resistance. Diabetes. 50:2069-2079.

21. Furler, S.M., Jenkins, A.B., Strolein, L.H., and Kraegen, E.W. 1991. In vivo location of the rate-limiting step of hexose uptake in muscle and brain tissue of rats. Am. J. Physiol. 261:E337-E347.

22. Matthew, D.R., et al. 1985. Homeostasis model assessment: insulin resistance and $\beta$-cell function from fasting plasma glucose and insulin concentrations in man. Diabetologia. 28:412-419.

23. Ockenga, J., Widjaja, A., Holtmannspötter, M., Schmidt, R.E., and Brabant, G. 1998. Bound leptin is regulated by tumor necrosis factor- $\alpha$ ind HIV-infected patients: a potential mediator of wasting? AIDS. 12:223-224.

24. Schmidt, H.H.-J., et al. 1999. Lipid evaluation in HIV-1 positive patients treated with protease inhibitors. Antivir. Ther. 4:163-170.

25. Caron, M., et al. 2001. The HIV protease inhibitor indinavir impairs sterol regulatory element-binding protein-1 intranuclear localization, inhibits preadipocyte differentiation, and induces insulin resistance. Diabetes. 50:1378-1388.

26. Murata, H., Hruz, P.W., and Mueckler, M. 2000. The mechanism of insulin resistance caused by HIV protease inhibitor therapy. J. Biol. Chem. 275:20251-20254

27. Cotes, H.C.F., et al. 2002. Changes in mitochondrial DNA as a marker of nucleoside toxicity in HIV-infected patients. N. Engl. J. Med. 346:811-820.

28. Brinkman, K., Smeitink, J.A., Romijn, J.A., and Reiss, P. 1999. Mitochondrial toxicity induced by nucleoside-analogue reverse-transcriptase inhibitors is a key factor in the pathogenesis of antiretroviral-therapyrelated lipodystrophy. Lancet. 354:1112-1115.

29. Sinnwell, T.M., et al. 1995. Metabolic abnormalities in skeletal muscle of patients receiving zidovudine therapy observed by $31 \mathrm{P}$ in vivo magnetic resonance spectroscopy. J. Clin. Invest. 96:126-131.

30. Wilson, J.E. 1995. Hexokinases. Rev. Physiol. Biochem. Pharmacol. 126:65-198.

31. van der Valk, M., et al. 2001. Increased risk of lipodystrophy when nucleoside analogue reverse transcriptase inhibitors are included with protease inhibitors in the treatment of HIV-1 infection. AIDS. 15:847-855.

32. Nolan, D., John, M., and Mallal, S. 2001. Antiretoviral therapy and the lipodystrophy syndrome, part 2: concepts in aetiopathogenesis. Antivir. Ther. 6:145-160.

33. Pan-Zhou, X.-R., et al. 2000. Differential effect of antiretroviral nucleoside analogs on mitochondrial function in HepG2 cells. Antimicrobial Agents Chemother. 44:496-503.

34. Lenhard, J.M., et al. 2000. HIV protease inhibitors block adipogenesis and increase lipolysis in vitro. Antiviral Res. 47:121-129.

35. Kahn, B.B., and Flier, J.S. 2000. Obesity and insulin resistance. J. Clin. Invest. 106:473-481.

36. Shulman, G. 2000. Cellular mechanisms of insulin resistance. J. Clin. Invest. 106:171-176.

37. Boden, G. 1997. Role in fatty acids in the pathogenesis of insulin resistance and NIDDM. Diabetes. 46:3-10.

38. Thomson, A.L., and Cooney, G.J. 2000. Acety-CoA inhibition of hexokinase in rat and human skeletal muscle is a potential mechanism of lipidinduced insulin resistance. Diabetes. 49:1761-1765.

39. Wiesenthal, S.R., et al. 1999. Free fatty acids impair hepatic insulin extraction in vivo. Diabetes. 48:766-774.

40. Bavenholm, P.N., Pigon, J., Oestenson, C.-G., and Efendic, S. 2001. Insulin sensitivity of suppression of endogenous glucose production is the single most important determinant of glucose tolerance. Diabetes. 50:1449-1454.

41. van der Valk, M., et al. 2001. Lipodystrophy in HIV-1 positive subjects is associated with insulin resistance in multiple metabolic pathways. AIDS. 15:2093-2100.

42. Bertoldo, A., et al. 2001. Kinetic modeling of [18F]FDG in skeletal muscle by PET: a four-compartment five-rate-constant model. Am. J. Physiol. Endocrinol. Metab. 281:E524-E536.

43. Reinhardt, M., et al. 1999. Quantification of glucose transport and phosphorylation in human skeletal muscle using FDG PET. J. Nucl. Med. 40:977-985.

44. Mynarcik, D.C., McNurlan, M.A., Steigbigel, R.T., Fuhrer, J., and Gelato, M.C. 2000. Association of severe insulin resistance with both loss of limb fat and elevated serum tumor necrosis factor receptor levels in HIV lipodystrophy. J. Acquir. Immune Defic. Syndr. 25:312-321. 